\title{
Verification of anti-fatigue effect of anserine by angle fatigue indicator based on median frequency changes of electromyograms
}

\section{Hirohisa Kishi $^{1,2}$, Daiki Kubomura ${ }^{3}$, and Toshifumi Sugiura ${ }^{4}$}

${ }^{1}$ Graduate School of Electronic Science and Technology, Shizuoka University, Hamamatsu, 432-8011, Japan; ${ }^{2}$ Department of Electronic and Information Systems Engineering, Polytechnic University, Kodaira, 187-0035, Japan; ${ }^{3}$ Yaizu Suisankagaku Industry, YSK, Yaizu, 425-8570, Japan; ${ }^{4}$ Research Institute of Electronics, Shizuoka University, Hamamatsu, 432-8011, Japan

*Corresponding authors: Hirohisa Kishi, Department of Electronic and Information Systems Engineering, Polytechnic University, Kodaira, 187-0035, Japan; ${ }^{2}$ Toshifumi Sugiura, Research Institute of Electronics, Shizuoka University, 432-8011, Japan

Submission Date: June 28, 2013; Acceptance Date: October 14, 2013; Publication Date: October 15,2013

Keywords: anserine, muscle fatigue, electromyogram, median frequency power, MDF angle

\section{BACKGROUND}

The city of Okinawa is known as a society of longevity where people enjoy katsuo (bonito) miso soup. This traditional food is used to combat physical fatigue, illness, and mental stress $[1,2]$. In Europe and China, chicken soup is also believed to have helped people recover from respiratory disorders, fatigue, frustration, anxiety, and other physical and mental conditions [3-5]. In addition to various amino acids, anserine is abundant in the skeletal muscles of fish and chicken, and is a common ingredient in miso soup. There are many reports on the bioactive properties of anserine, particularly its anti-fatigue effect [6-8].

Anserine is a dipeptide which is composed of $\square$-alanine and $\square$-methy-L-histidine and is synthesized in skeletal muscles in vertebrates [9]. It is abundant in the flesh of avian species and in a widerange of fish such as bonito and tuna, while carnosine, which is composed of $\square$-alanine and L-histidine, is abundant in terrestrial mammals, including humans. These substances are reported to have various bioactive functions, such as operating as a $\mathrm{pH}$ buffer [10], activation of 
myosin ATPase [6], promotion of the glycolytic reaction [11], antioxidative effect [12], chelative action [13], as well as control of blood sugar [14].

Even though a number of studies have reported the anti-fatigue action of anserine, such as the forced swimming test in mice [15], an in vitro muscle fiber investigation [7], and the hormonal response to resistance exercise [16], direct verification in human subjects is not that straight forward and insufficient evidence exists [17]. Reasons for differences and variation include great differences among individuals and even variation in the physical condition one of a single subject. The aim of this study was to verify the anti-fatigue effect of anserine in human subjects as directly as possible.

\section{METHODS}

Subjects: Subjects were 17 healthy male volunteers $(35.5 \pm 5$ yr., $75.5 \pm 5.0 \mathrm{~kg})$ who were requested to get sufficient sleep, and to refrain from consuming alcohol, coffee and other stimulating beverages, and from smoking for $18 \mathrm{~h}$ prior to the start of the experiment. On the morning of the experiment, subjects skipped breakfast. No participant was using drugs or medication. We excluded female volunteers because the hormone balance in female generally fluctuates enormously compared to male hormone levels, and exercise loads in this experiment seemed to be hard for them. The study was performed in accordance with the 1964 Declaration of Helsinki, and was approved by the Shizuoka University Research Ethics Committee. Participants were only enrolled in the study after giving their informed consent.

Test substance: Edible-grade anserine was purified from fish extract with food-grade reagents. The muscle of skipjack tuna $(800 \mathrm{~g})$ was boiled in $1.5 \mathrm{~L}$ of tap water for $1 \mathrm{~h}$, and the broth was collected and filtered through no. 2 paper (Advantec Toyo Kaisha Ltd., Tokyo, Japan). It was then ultrafiltered to remove macromolecules, such as proteins, through a CF-30 membrane (Amicon, Beverly, MA), desalted with an NTR-729 reverse osmotic membrane (Nitto Denko Co., Tokyo, Japan), and purified on an ion-exchange column of cation-exchange resin Dowex 50-X4. After lyophilization, anserine powder was obtained and analyzed for anserine content by high-performance liquid chromatography (HPLC).

Exercise protocols: The study followed a double-blind approach. The subjects performed the isometric exercise tolerance test (ETT) on the rectus femoris muscle. They maintained their right or left leg in a horizontal position (unilateral leg extension) in a sitting posture. Each subject 
performed the same exercise twice on a different day at least one week apart. Subjects consumed anserine or water (control). Anserine was dissolved in warm water $\left(30^{\circ} \mathrm{C}, 200 \mathrm{ml}\right)$ at a dosage based on body mass $(11 \mathrm{mg} / \mathrm{kg})$. Most subjects were not able to notice the difference in taste between the water that contained anserine and the control. The time sequence after the intake of anserine or water was: 20 min rest (Rest-1), first ETT for 5 min (Ex-1), 15 min rest (Rest-2), second ETT for 3 min (Ex-2), 5 min rest (Rest-3). The order of anserine or water intake was randomly changed from subject to subject. Figure 1 shows a visual representation of the exercise (a) and its time sequence (b).
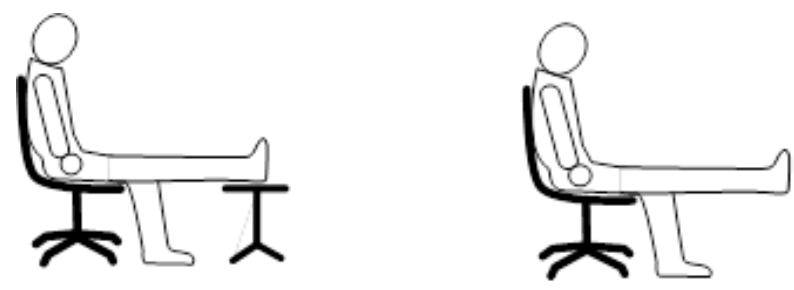

(a) Isometric exercise tolerance test (ETT).

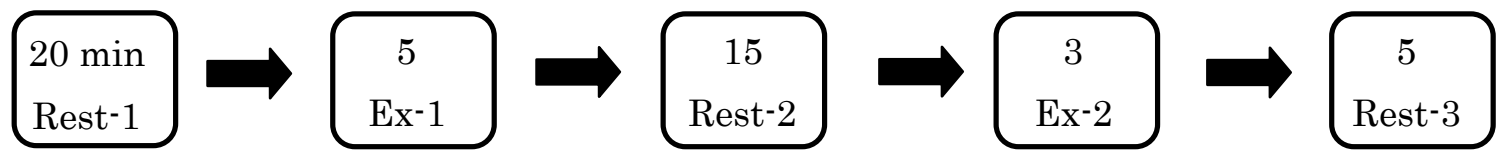

(b) Time sequence of ETT.

Figure 1: Isometric exercise tolerance test (a) and its time sequence (b). A subject was asked to maintain his right or left leg in a horizontal position (unilateral leg extension) in a sitting posture. Each subject performed the same exercise twice on a different day at least one week apart.

Measurement of electromyogram: An electromyogram was placed on the surface of the rectus femoris muscle and XYZ was measured with disposable electrodes (F-150S, Nihon Koden) attached to an amplifier (EMG-021, Harada Electronic Industry Ltd.) and fed to a computer through an A/D converter (NI -USB6218, 16-bit, National Instruments Co.) at $1 \mathrm{kHz}$.

Analytical methods: Fourier analysis was performed on the EMG data (Ex-1 and Ex-2) with a Hanning window $(10 \mathrm{sec})$. The data over a frequency range of 21-214 Hz was targeted for further analysis. Median frequencies (MDFs), which are considered to show muscle fatigue [18] and described by eq. (1), were obtained for Ex-1 and Ex-2. 


$$
\int_{0}^{M D F} P(f) d f=\int_{M D F}^{\infty} P(f) d f
$$

(1) Where P(f) and MDF are the power spectral density and median frequency, respectively. A regression curve was calculated from changes in MDF during Ex-1 and Ex-2. Since a measure of steepness of the regression curve indicates the degree of muscle fatigue, we compared the difference of the two slopes (Ex-1 and Ex-2), or the angle between these slopes (MDF angle). The MDF angle was used as an index of fatigue. In other words, a larger angle indicated greater fatigue. This angle was obtained by eq. (2).

$$
\text { Angle }=\cos ^{-1} \theta=\frac{(1 \times 1)+\left(G_{E x 1} \times G_{E x 2}\right)}{\sqrt{1^{2}+G_{E x 1}^{2}} \times \sqrt{1^{2}+G_{E x 2}^{2}}}
$$

(2) Where $G_{E x 1}$ and $G_{E X 2}$ are the slopes of the regression curves during Ex-1 and Ex-2, respectively.

Statistical analysis: Differences in the change in angle between anserine and water (control) intake were evaluated by a paired $t$-test. The significance was defined as $\mathrm{p}<0.01$.

\section{RESULTS}

An example of the measured EMG is shown in Figure 2. Two large amplitude regions correspond to the first (Ex-1) and the second (Ex-2) exercise. Figure 3 shows the changes of MDF for Ex-1 (a) and Ex-2 (b). FFT was performed every $10 \mathrm{sec}$ during the exercise. The horizontal axis is the elapsed time (sec) and the vertical axis is the MDF (Hz). Although MDF gradually decreased over time, MDF in Ex-2 fell at a slower pace than that in Ex-1. Regression lines (in red) are also drawn in the figure. The difference between these two slopes ( $\left.\square_{\mathrm{Ex}-1}-\square_{\mathrm{Ex}-2}\right)$ was obtained as a differential angle by eq. (2).

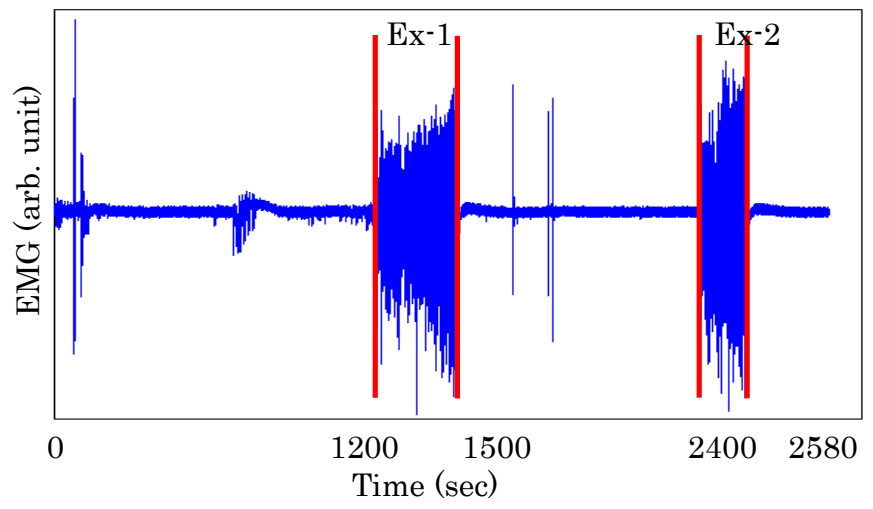

Figure 2: Electromyogram during the first (Ex-1) and second (Ex-2) exercise period. 


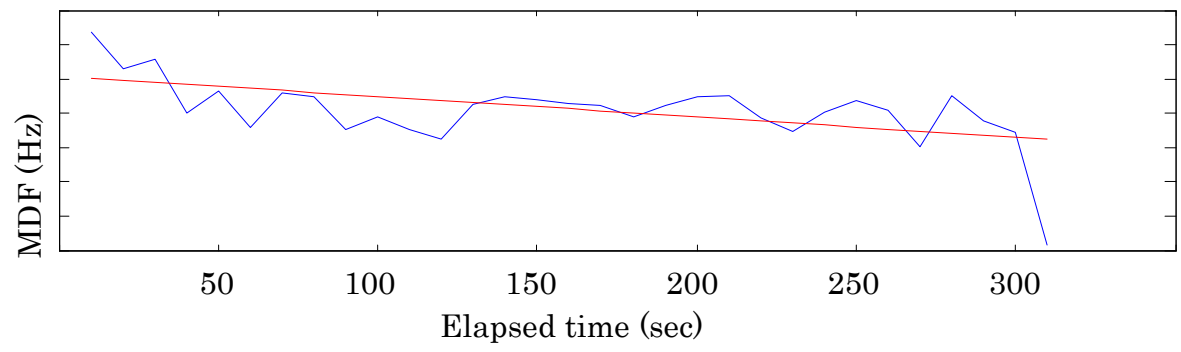

(a) MDF change and slope during the first exercise

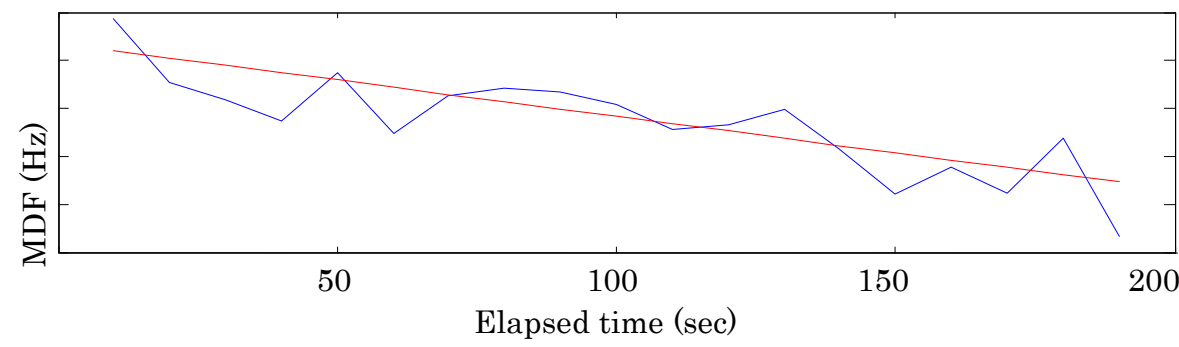

(b) MDF change and slope during the second exercise

Figure 3: Median frequency changes during the first (a) and the second (b) exercises. Regression curves (MDF slopes) are also drawn in red.

Figure 4 compares the angles obtained by eq. (2) for anserine and water intake. Most angles for Ex-1 and Ex-2 are negative because the slopes for Ex-2 are steeper than those for Ex-1 both in anserine and water (control) intake, which suggests the anti-fatigue effect of anserine.

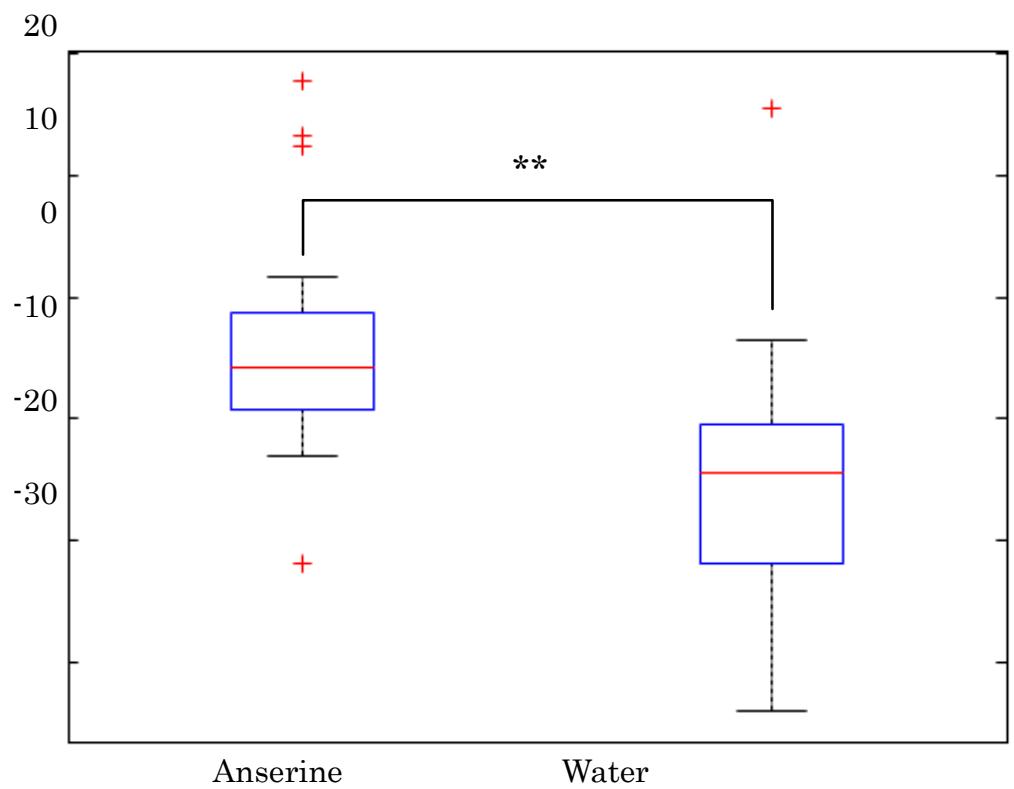

Figure 4: Median trequency changes during the first (a) and the second (b) exercises. Regression curves are also drawn. The MDF in Ex-2 (b) decreases faster than that in Ex-1 (a). n=17, **p<0.01. 


\section{DISCUSSION}

There are several ways to measure muscle fatigue in humans, such as with lactate $[3,20]$, muscle sound [22], proton ion [25], oxygen saturation [21], ATPase [6], or by other means. However, in other studies, these measurements did not directly show the anti-fatigue effect in human subjects. Observing the reduction in median frequency or mean power frequency of electromyogram is another and conventional way to investigate the muscle fatigue $[18,23,26]$. However, as far as we know, this paper is an original study which applied the EMG to the verification of anti-fatigue effect of anserine in human subjects. Moreover, we added a new twist to the measurement protocol.

In this study, each subject performed ETT twice in a day with anserine or placebo and again performed the same ETT twice on a different day with placebo or anserine at least one week apart. The second ETT in a day was performed at 40 min after intake because blood anserine concentration reaches its maximum at that time [24]. The MDF slope at the second exercise was usually larger than that in the first, which implies that fatigue worsened in the second test. The error which stems from the daily variations in the physical condition of a subject can be minimized by comparing the differences of the MDF angles obtained on different days. Our results indicate that anserine has the effect of reducing muscle fatigue in humans. Although this is a preliminary result, to our knowledge, it is the first direct demonstration of the anti-fatigue effect of anserine in humans based on EMG.

The underlying mechanisms responsible for the anti-fatigue effect of anserine are complicated [19] and may be a mixture of many actions, such as the reduction of plasma lactate [3], an anti-oxidant effect [19], activation of ATPase [6], promotion of glycolytic reactions [12], proton buffering action [25], etc. We think that all these actions seem to be beneficial to a prolonged duration of exercise, though unfortunately, a more rigorous assessment is beyond our capability.

The dose of anserine used in this study was $11 \mathrm{mg} / \mathrm{kg}$. We plan to increase the dose up to $1-2 \mathrm{~g} / \mathrm{kg}$ to investigate the effects on autonomic nervous activities as well as on blood pressure. 
Limitations: The mechanisms underlying muscle fatigue are complicated, depending on the subject's physical fitness, age, gender, and the type of exercise. The anti-fatigue effects of anserine for females, other ages and type of exercise need to be studied.

\section{CONCLUSIONS}

We compared the difference of MDF slopes of EMG or the angle between these slopes during two ETTs. Greater angles indicate increased fatigue. The data indicate that anserine can alleviate muscle fatigue in humans. The exact functional mechanism needs to be clarified. An investigation on the effect of anserine on autonomic nervous activities is currently in progress.

Abbreviations: EMG, electromyogram; ETT, exercise tolerance test; MDF, median frequency

\section{Competing interests}

The authors declare that they have no competing interests with anyone or with any organizations.

\section{Authors' Contributions}

All authors contributed to this study. Hirohisa Kishi (kishi@uitec.ac.jp) designed and conducted the study, performed the data analysis, including the statistical analysis, and drafted the manuscript. Daiki Kubomura (kubomura@yskf.co.jp) extracted and purified the anserine powder from the muscle of skipjack tuna. Toshifumi Sugiura (sugiura@rie.shizuoka.ac.jp) initiated the

study, supervised and co-designed the entire study, and also co-revised and edited the manuscript in its entirety.

\section{Acknowledgements and Funding}

The authors would like to thank all subjects who volunteered in this study and our colleagues in the laboratory for technical assistance. This study was supported by the Ministry of Education, Culture, Sports, Science \& Technology in Japan. 


\section{REFERENCES:}

1. Kuroda M, Ishizaki T, Maruyama T, Takatsuka Y, Kuboki T. Effect of dried-bonito broth on mental fatigue and mental task performance in subjects with a high fatigue score. Physiology \& Behavior 2007; 92 (5): 957-962.

2. Umeki Y, Hayabuchi H, Hisano M, Kuroda M, Honda M, Ando B, Ohta M, Ikeda M. The effect of the dried-bonito broth on blood pressure, 8-hydroxydeoxyguanosine (8-OHdG), an oxidative stress marker, and emotional states in elderly subjects. Journal of Clinical Biochemistry and Nutrition 2008; 43 (3): $175-184$.

3. Lo HI, Tsi D, AC Tan, Wang SW, Hsu MC. Effects of post-exercise supplementation of chicken essence on the elimination of exercise-induced plasma lactate and ammonia. Chinese Journal of Physiology 2005; 48: 187-192.

4. Li YF, He RR, Tsoi B, Kurihara H. Bioactivities of chicken essence. Journal of Food Science 2012; 77(4): R105-10, doi: 10.1111/j.1750-3841.2012.02625.x.

5. Caroline NL, Schwartz H. Chicken soup rebound and relapse of pneumonia: Report of a case. Chest 1975; 67 (2): 215-216.

6. Avena RM, Bowen WJ. Effects of carnosine and anserine on muscle adenosine triphosphatases. The Journal of Biological Chemistry 1969; 244 (6), 1600-1604.

7. Everaert I, Stegen S, Vanheel B, Taes Y, Derave W. Effect of beta-alanine and carnosine supplementation on muscle contractility in mice. Medicine \& Science in Sports \& Science 2013; 45 (1), 43-51.

8. Derave W, Özdemir MS, Harris RC, Pottier A, Reyngoudt H, Koppo K, Wise JA, Achten E. B-Alanine supplementation augments muscle carnosine content and attenuates fatigue during repeated isokinetic contraction bouts in trained sprinters, Journal of Applied Physiology 2007; 103, 1736-1743, doi:10.1152/japplphysiol.00397.2007.

9. Suzuki T, Hirano T, Suyama M. Free imidazole compounds in white and dark 
muscles of migratory marine fish, Comparative Biochemistry and Physiology B 1987; 87 (3), 615-619.

10. Ave H, Dobson GP, Hoeger U, Parkhouse WS. Role of histidine-related compounds to intracellular buffering in fish skeletal muscle, American Journal of Physiology 1985; 249, 449-454.

11. Davey CL. The effects of carnosine and anserine on glycolytic reactions in skeletal muscle, Archives of Biochemistry and Biophysics 1960; 89, 296-302.

12. Boldyrev AA, Bulygina E, Leinsoo T, Pertrushanko I, Tsubone S, Abe H. Protection of neuronal cells against reactive oxygen species by carnosine and related compounds, Comparative Biochemistry and Physiology B 2004; 137, 81-88.

13. Miller DJ, O’Dowd A. Vascular smooth muscle actions of carnosine as its zinc complex are mediated by histamine $\mathrm{H}(1)$ and $\mathrm{H}(2)$ receptors, Biochemistry 200; 65 (7), 938-948.

14. Kubomura D, Matahira Y, Nagai K, Niijima A. Effect of anserine ingestion on the hyperglycemia and autonomic nerves in rats and humans, Nutritional Neuroscience 2010; 3 (3), 123-128.

15. Kihlstöm M, Ojala J, Salminen A. Decreased level of cardiac antioxidants in endurance-trained rats, Acta Physiologica Scandinavica 1989, 135 (4); 549-554.

16. Goto K, Maemura H, Takamatsu K, and Ishii N. Hormonal responses to resistance exercise after ingestion of carnosine and anserine, Journal of Strength \& Conditioning Research 2011; 25(2), 398-405, doi: 10.1519/JSC.0b013e3181bac43c.

17. Lindberg F, Öhberg F, Brodin LÅ, Gönlund C. Assessment of intramuscular activation patterns using ultrasound M-mode strain, Journal of Electromyography and Kinesiology 2013; http://dx.doi. org/10.1016/j.jelekin.2013.02.011.

18. Dingwell JB, Joubert JE, Diefenthaeler F, Trinity JD. Changes in muscle activity 
and kinematics of highly-trained cyclists during fatigue, IEEE Transactions on Biomedical Engineering 2008; $55 \quad$ (11), 2666-2674, doi:10.1109/TBME.2008.2001130.

19. Culbertson JY, Kreider RB, Greenwood M, Cooke M. Effects of beta-alanine on muscle carnosine and exercise performance. A review of the current literature, Nutrients 2010; 2, 75-98, doi.10. 3390/nu2010075.

20. Finsterer J. Biomarkers of peripheral muscle fatigue during exercise, BMC Musculoskeletal Disorders 2012; 13, pp.218-230.

21. Cannon DT, White AC, Andriano MF, Kolkhorst FW, Rossiter HB. Skeletal muscle fatigue precedes the slow component of oxygen uptake kinetics during exercise in humans, Journal of Physiology 2011; 589.3, 727-739.

22. Malek MH, Cobum JW. The utility of electromyography and mechanomyography for assessing neuromuscular function: a noninvasive approach, Physical Medicine and Rehabilitation Clinics of North America 2012; 23 (1), 23-32, doi: 10.1016/j.pmr.2011.11.005.

23. Camic CL, Housh TJ, Zuniga JM, Hendrix R, Bergstrom HC, Traylor DA, Schmidt RJ, Johnson GO: Electromyographic and mechanomyographic responses across repeated maximal isometric and concentric muscle actions of the leg extensors, Journal of Electromyography and Kinesiology 2013; 342-348.

24. Kubomura D, Matahira Y, Masui A, Matsuda H: Intestinal absorption and blood clearance of L-histidine-related compounds after ingestion of anserine in humans and comparison to anserine-containing diets, Agricultural and Food Chemistry $2009 ; 57,1781-1785$.

25. Abe $\mathrm{H}$ : Role of histidine-related compounds as intracellular proton buffering constituents in vertebrate muscle, Biochemistry 2000; 7, 757-795.

26. TW Beck, TJ Housh, GO Johnson, JT Cramer, JP Weir, JW Coburn, MH Malek: Does the frequency content of the surface mechanomyographic signal reflect 
motor unit firing rates? A brief review, Journal of Electromyography and Kinesiology 2007; 17, 1-13. 